Kalpa Publications in Computing
Volume 4, 2018, Pages 94-109
28th International Workshop on
Principles of Diagnosis (DX'17)

\title{
Autonomous vehicle traction subsystem modeling and diagnosis using BG-PCs*
}

\author{
Carlos J. Alonso-González ${ }^{1}$, Anibal Bregon ${ }^{1}$, Belarmino Pulido ${ }^{1}$, \\ Matias Nacusse ${ }^{2,3}$, and Sergio Junco ${ }^{2,3}$ \\ ${ }^{1}$ Universidad de Valladolid, Valladolid, Spain \\ calonsodinfor.uva.es, anibaleinfor.uva.es, belareinfor.uva.es \\ 2 Laboratorio de Automatizacion y Control, Facultad de Ciencias Exactas, Ingenieria y Agrimensura \\ Universidad Nacional de Rosario, Argentina. \\ nacussedfceia.unr.edu.ar, sjuncolfceia.unr.edu.ar \\ 3 CONICET: Consejo Nacional de Investigaciones Cientificas y Tecnicas, Argentina
}

\begin{abstract}
Fault diagnosis is an essential part in the Health Management of autonomous vehicles. Within these vehicles the traction subsystem is a critical component, especially in those exploring planetary surfaces. Recent advances in brushless DC motors has raised the interest in new models and control configurations to integrate them in those vehicles due to their low energy consumption high torque/mass ratio and low maintenance requirements. In this work we develop a full Bond Graph model of this subsystem, including the brushless motor and the control blocks needed for proper and efficient operation. These models will allow us to perform fault diagnosis with Bond Graph Possible Conflicts as the unifying formalism. We derive the Bond Graph-Possible Conflicts of the system, discussing the viability of the proposal.
\end{abstract}

\section{Introduction}

There is an increasing interest in sending autonomous exploration vehicles to the space, and in doing so in an economical and practical way, emphasizing standard components [21]. It seems obvious that these systems will require an automatic fault diagnosis and recovery system.

Locomotion is one of the most important subsystems in those vehicles, and being able to diagnosis the electromechanical traction system is a must. In these subsystems wheel engines play an important role.

The small amount of energy demanded and its low maintenance requirements have made Brushless DC motors (BLDCM) an ideal candidate. But there are limited references about how to diagnose these kinds of engines, because most of the research is devoted to induction motors. For that reason we propose to explore model-based diagnosis on a BLDCM used in the traction system of an autonomous rover.

${ }^{*}$ This work has been supported by the Spanish Ministery of Economy and Competitiveness under grant DPI2013-45414-R. Authors would like to thank the collaboration from GMV Aerospace for their technical support related to rover testbed. 
There are several proposals in the literature about the components in a traction subsystem for autonomous vehicles[11, 28]. All of them agree in the main components. We have selected one of the publically available designs together with open access specification of a typical BLDCM motor [17]. In the paper we include the Bond Graph models of the motor and its variables transformation, together with detailed block diagrams of the control and electronic needed for proper and efficient operation.

The rover is a hybrid and distributed system, hence we look for a model-based diagnosis approach as general as possible. In this work we focus on the traction system for a given control configuration and propose a Bond-Graph model. We analyze how model-based diagnosis with BG-PCs can handle Health Management of these vehicles finding out the actual set of BG-PCs given the proposed model and its set of observations.

The organization of this article is as follows: first we introduce the case study by presenting fundamental issues related to BLDCM; second, we provide the block diagram of the complete subsystem, providing aftwerwards all the Bond Graphs to model the motors, the gear and the load components. Then we summarize the BG-PC approach for model-based diagnosis. Later on we present the BGPCs of the systems. Finally we illustrate fault detection and isolation capabilities for the BG-PCs with simulation data, drawing some conclusions.

\section{Case Study - Traction Subsystem in an autonomous vehicle}

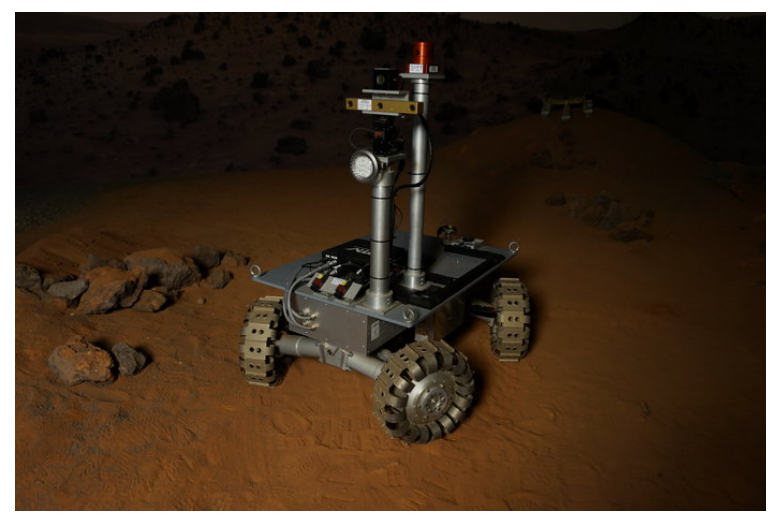

Figure 1: Example of autonomous vehicle: Lunar Rover Model (LRM), RAT's Physical Flight Segment (Image (c) European Space Agency).

The purpose of our project is to develop a generic framework for diagnosis and prognosis of hybrid and distributed systems. We have studied different proposals as an starting point for our project requirements, among them the European Space Agency (ESA) lunar rover model [11, 16, 17], together with different autonomous vehicles such as the RobuCar testbed [15] or the NASA autonomous testbed for diagnosis and prognosis [1,2]. All of them share similar design in terms of the traction system: 4/6 wheels and motors controlled by different current/position/velocity control loops, bearing mechanisms, together with a payload, etc.

In the traction system there are different options regarding the engine selected to drive the vehicle and which magnitude is used to control the motors. Since there is an increasing interest in using Brushless DC motors (BLDCM) due to their small maintenance requirements and its low energy demand, we have decided to analyze one architecture, which could fit within any of those previous testbeds, organized around a BLDCM. The main reason is the lack of studies about these motors due to the massive 


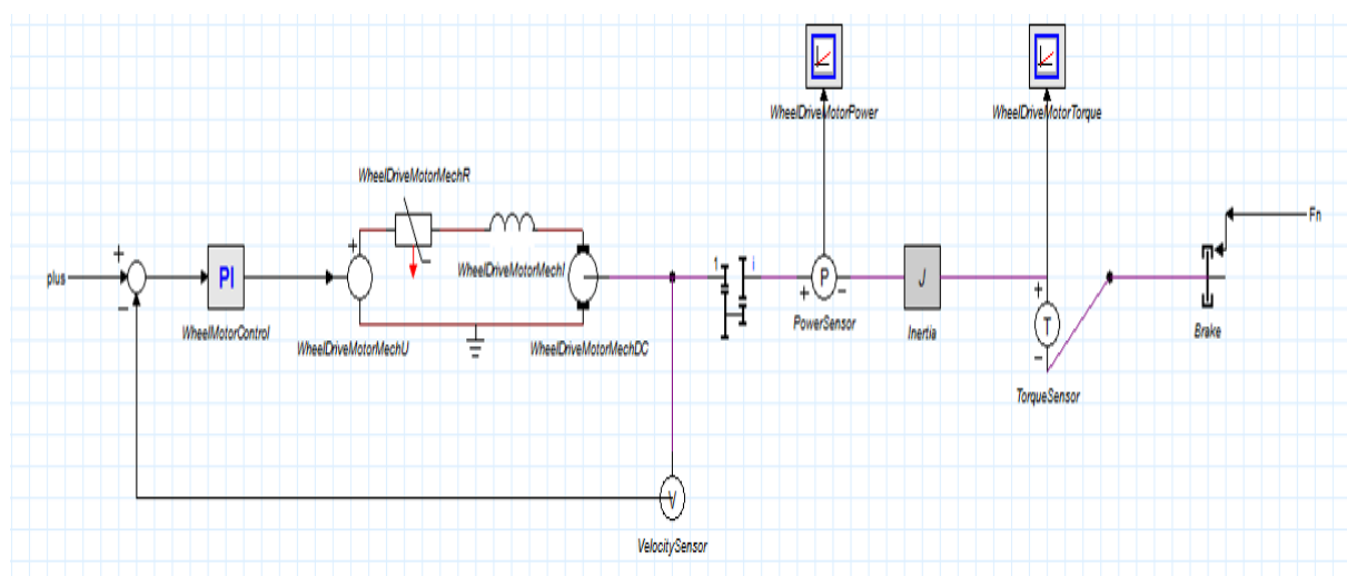

Figure 2: Rover Autonomy Testbed developed for ESA.

presence of designs including induction motors.

We have decided to adapt a proposal made by Medina et al. [17] (see Figure 2). The traction system is centered around a speed controller acting upon a voltage source. Given the limited amount of electrical power available in an autonomous rover, moving to a BLDCM, more efficient and robust than the series DC motor used in [17], seems to be a natural improvement. The problem we want to discuss at the workshop is a new design, model-based diagnosis oriented, with a unique modelling framework.

Regarding the BG modelling, we have followed Junco and Donaire [13] with his proposal for the BLDCM motor, including speed and torque control. However, we provide a deeper detail of the software/electronic configuration needed to feed the real stator, with insights usually not found in the literature.

Finally we have studied different proposals for the gear and load parts available in the mechatronics literature [26, 18], and finnaly adapted the solution by Loureiro et al. [15] for the gear and load parts because they are generic enough and easily modifiable if necessary.

In the rest of the paper we show the main features of these motors and how they can be modelled using Bond-Graph models. We then use such models to compute minimal submodels (BG-PCs) which can be used for efficient diagnosis and prognostics.

\section{Brushless Permanent Magnet DC motors}

Brushless Permanent Magnet DC motors have become a popular option for Rover drive motors [21] because of its high torque/mass ratio together with a low maintenance requirements and high robustness due to the lack of mechanical brushes, [27], [3].

Nevertheless, this kind of motors need to be commanded by a complex electronic, compared to brushed DC motors, because the stator currents have to be carefully switched to make the rotor turn and deliver useful torque. There are three basic approaches to stator currents commutation: trapezoidal commutation, sinusoidal commutation and Field Oriented Control, FOC. Field Oriented Control provides a smooth motion at low speeds and efficient operation at high speeds, combining the best of trapezoidal and sinusoidal commutation, [9]. Hence, in this paper we consider FOC of the brushless DC motor. FOC requires to control the motor in the so called $d-q$ reference frame [13]. This formalism has the 
advantage that controllers work with DC currents, independently of the sinusoidal signal that feed the stator coils, [9], [14].

\subsubsection{The general reference frame $(d, q, 0)$ - coordinate system}

Even under the usual simplifying assumptions, like perfect symmetry, perfect sinusoidal distribution of magnetomotive force and absence of magnetic saturation and hysteresis, the models of electrical machines are quite involved if written in machine variables (i.e., stator voltages and currents). The so-called general reference-frame theory comprises most of the changes of variables developed over the years in the theoretical analysis of electrical machines [13], [14]. Some of the advantages of the reference frame theory are:

- the number of voltage equations is smaller, as well as their complexity

- when properly aligned with the rotor and stator coordinate system, the time varying stator variables, voltages, fluxes and currents, become stationary

- it allows for simple control loops, that operate directly on the stationary $(d, q)$ variables, independently of the stator voltages frequency

Nevertheless, for the scope of this paper, the $(d, q)$ reference frame can be interpreted as a rotational coordinate transformation, [13].

Figure 3 shows a cross section on a plane transversal to the machine's longitudinal axis of rotation, $o$. The rotor consists of a permanent magnet while the stator consists of three coils for a three phase stator. The coordinate system $(x, y, o)$ is fixed to the stator.

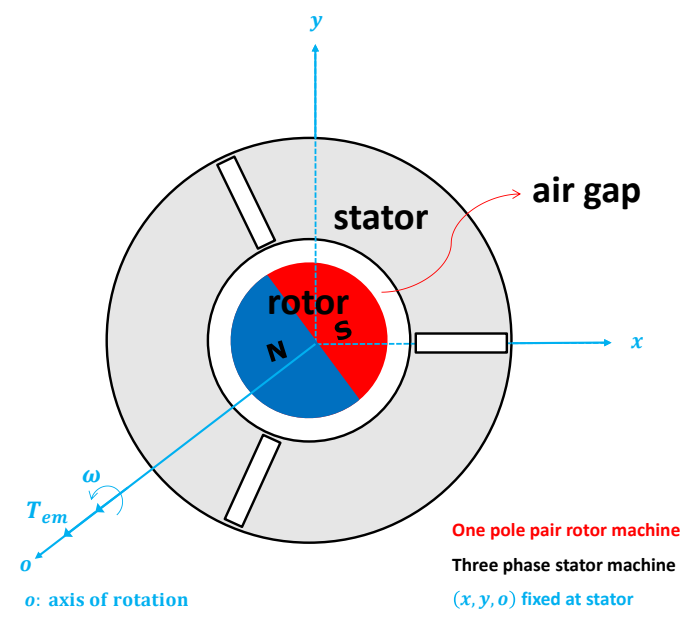

Figure 3: Cross section of a BLDCM

Figure 4 shows the stator electrical axes, $(a, b, c), 120^{\circ}$ apart from each other, with its $a$-axis aligned with the $x$-axis. The orthogonal $(d, q)$ system rotates at an arbitrary speed $\omega_{F}(t)$, and its instantaneous position is given by the angle $\rho(t)=\int \omega_{F}(t)$ between the $d$ - and $x$ - axes. A one pole pair rotor, aligned to the reference frame, that is, with instantaneous electrical position $\theta_{r}=\rho$, is shown in a red rectangle. For a one pole pair rotor machine, with its magnetic axis aligned to the $d$-axis, the actual rotor speed is 
the electrical rotor speed, $\omega=\omega_{r}$, and the rotor instantaneous angular position $\theta=\theta_{r}=\int \omega_{F}(t)$. For a general machine with $n_{p}$ pairs of poles, $\omega=\omega_{r} / n_{p}$.

The general reference frame is obtained augmenting the $(d, q)$ to a $(d, q, 0)$ coordinate system. The $(x, y, 0)$ coordinate systems is referred to as the stationary coordinate system.

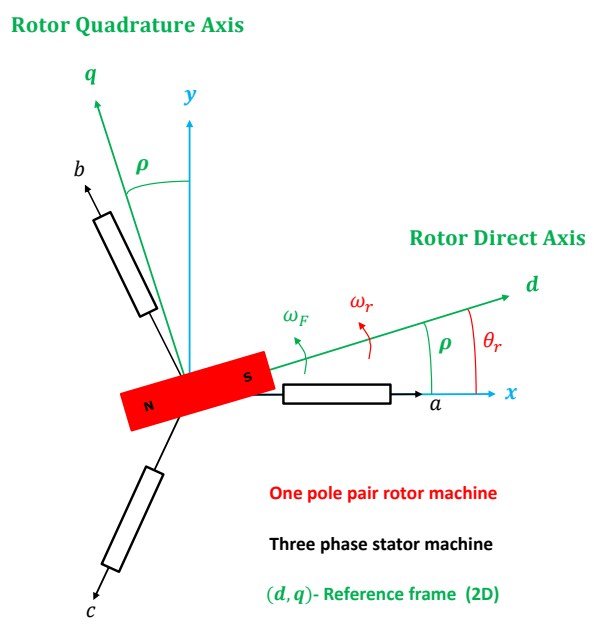

Figure 4: Reference frame for a BLDCM with rotor aligned to reference frame

\subsubsection{Coordinate transformation}

All stator variables are transformed to a common reference frame. Let $\left\{u_{a s}, u_{b s}, u_{c s}\right\}$ be a generic triplet of stator variables (i.e. currents, voltages, fluxes). This triplet is transformed into another triplet in the reference frame: $\left\{u_{d s}, u_{q s}, u_{0 s}\right\}$, according to the transformation $M_{s}=M(\rho(t))$.

$$
\begin{aligned}
& M_{s}=\sqrt{\frac{2}{3}}\left[\begin{array}{ccc}
\cos \left(\theta_{r}\right) & \cos \left(\theta_{r}-\frac{2 \pi}{3}\right) & \cos \left(\theta_{r}+\frac{2 \pi}{3}\right) \\
-\sin \left(\theta_{r}\right) & -\sin \left(\theta_{r}-\frac{2 \pi}{3}\right) & -\sin \left(\theta_{r}+\frac{2 \pi}{3}\right) \\
\frac{1}{\sqrt{2}} & \frac{1}{\sqrt{2}} & \frac{1}{\sqrt{2}}
\end{array}\right] \\
& {\left[\begin{array}{l}
u_{d s} \\
u_{q s} \\
u_{0 s}
\end{array}\right]=M_{s}\left[\begin{array}{l}
u_{a s} \\
u_{b s} \\
u_{c s}
\end{array}\right]}
\end{aligned}
$$

The inverse matrix satisfies $M_{s}^{-1}=M_{s}^{T}$.

\subsubsection{Equivalent electrical circuit of a BLDCM}

Figure 5 shows the equivalent electrical circuit of a BLDCM, adapted from the equivalent circuit of a general synchronous machine found in [13]. There is additionally the fixed flux of the permanent magnet of the rotor, $\psi_{P M}$. Keep into account that if the reference frame $d$ axis is aligned with the axis on which the rotor magnet is making positive flux -the axis of the magnet for a two pole rotor, see Figure 4- the magnets produce flux on the $d$ axis only [14].

The inductances $L_{l s}$ are leaking inductances. The inductances $L_{m d, m q}$ are the magnetizing inductances of each axis, and they are equal for a round-rotor machine because of rotor symmetry. 


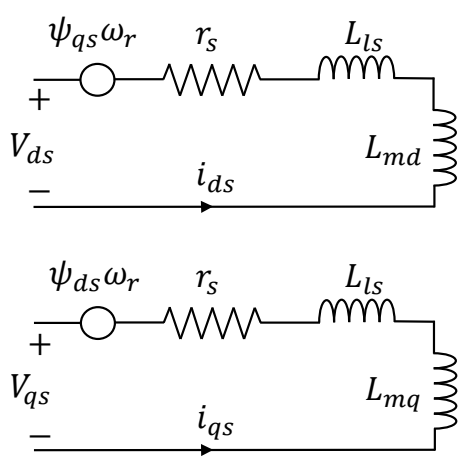

Figure 5: Equivalent electrical circuit of a one pole pair three phase stator BLDCM.

The electromagnetic torque is given by:

$$
T_{e m}=n_{p}\left(\psi_{d s} i_{q s}-\psi_{q s} i_{d s}\right)
$$

The fluxes are given by:

$$
\begin{gathered}
\psi_{d s}=\left(L_{l s}+L_{m d}\right) i_{d s}+\psi_{P M}=L_{d} i_{d s}+\psi_{P M} \\
\psi_{q s}=\left(L_{l s}+L_{m q}\right) i_{q s}=L_{q} i_{q s}
\end{gathered}
$$

\section{The Traction subsystem Bond Graph Model}

Bond Graph (BG) modeling approach is a domain-independent energy-based topological modeling language for physical systems [7, 23]. Several types of primitive elements are used to build BGs: storage elements (capacitors, C, and inductances, I), dissipative elements (resistors, R) and elements for energy transformation (transformers, TF, and gyrators, GY). There are also effort and flow sources (Se and Sf), which are used to define interactions between the system and the environment. Elements in a BG are connected by 0 or 1 junctions (representing ideal parallel or series connections between components, respectively). Each bond has associated two variables (effort and flow).

\subsubsection{Coordinate transformation using Bond Graphs.}

Given that the BG model of the BLDCM is developed in the $(d, q, 0)$ reference frame, but the stator has to be fed with sinusoidal voltages on the $(a, b, c)$ coordinate system, the matrixes $M_{s}$ and $M_{s}^{-1}$ are necessary to show the stator variables in the graph. Figure 6 shows the BG to transform the stator variables into reference frame variables, that can be fed into the BLDCM Bond Graph in the reference frame. 


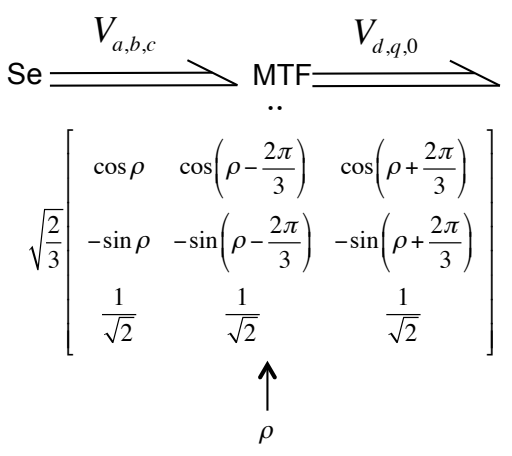

Figure 6: BG transformation to reference frame.

\subsubsection{The PWM block}

Pulse Width Modulation, PWM, is a well know technique to supply a variable voltage at a given frequency to some power demanding appliance from a DC source [12,14]. Because of its high efficiency, it is the common solution to transfer power from a DC source to a frequency driven load, like the stator of a BLDCM.

The input of the PWM module are the stator sinusoidal voltages, $V_{a}, V_{b}$ and $V_{c}$, computed from $V_{d}$ and $V_{q}$. The output of the PWM are the real signals injected at the stator windings, $V_{a_{p w m}}, V_{b_{p w m}}$, and $V_{c_{p w m}}$. See Figure 7 for a graphical description.

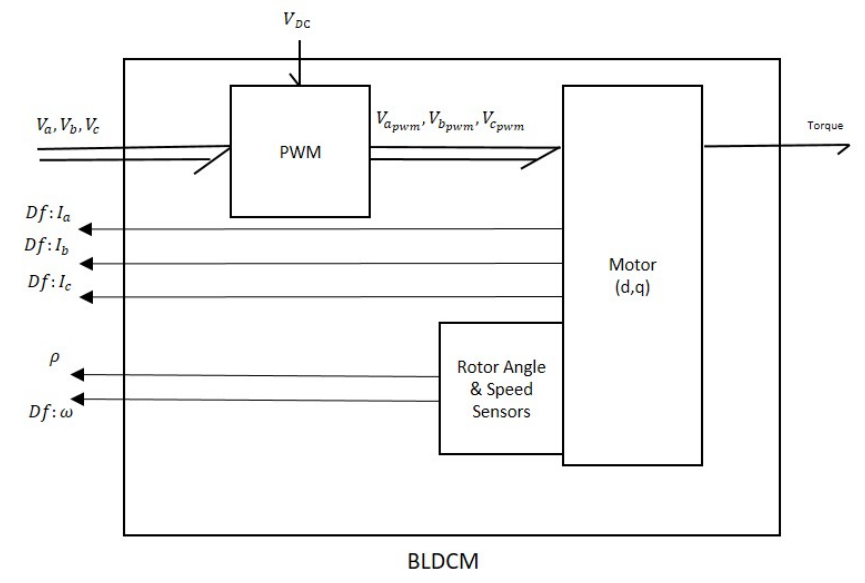

Figure 7: BLDCM block diagram.

\subsubsection{Bond Graph of a BLDCM}

The Bond Graph corresponding to the equivalent electrical circuit shown in Figure 5 is displayed in Figure 8. The original proposal comes from Junco and Donaire [13]. The sources associated to $V_{d}$ and $V_{q}$ are potentially controlled sources of voltage imposed on the motor. The source associated to the torque should be interpreted as a mechanical load. 


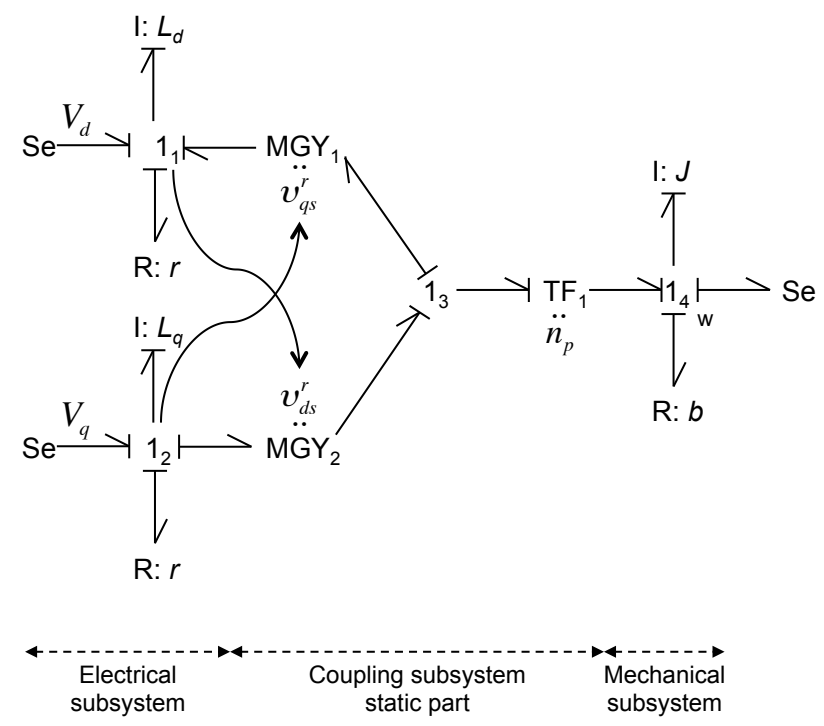

Figure 8: BG of a BLDCM in the reference frame.

\subsection{Traction subsystem Bond Graph diagram}

Once the system constitutive elements have been described, we can formulate different high level control laws. We have adapted the proposal of [9], commonly used elsewhere, where an external angular speed controller commands, in cascade configuration, the currents in the $(d, q)$ reference frame. Figure 9 shows a high level description of the speed controller-BLDCM-Wheel traction subsystem for one motorwheel pair.

For the sake of simplicity, we describe the current controllers as standard PI regulators. The practical implementation on a real planetary rover may require, however, much more complex control laws, including, for instance, feed forward compensations and current limiters.

The $I_{q}$ current is used to command the torque while the $I_{d}$ is used to weaken the flux in the $d$-axis. This scheme carries the $d$ current component close to zero. Since only the $q$ - current produce useful torque, this maximizes the torque efficiency of the system [9]. Simultaneously, reducing the $d$ current reduce the back electromotive force, responsible of a considerable portion of voltage drop, allowing the motor to accelerate quicker [10].

The outputs of the current controllers are the tensions on the reference frame. They have to be transformed into the stator voltages that feed the PWDM+BLDCM block. The real currents fed into the stator have to be measured and transformed back to the reference system to close the current loops.

The coordinate transformations require knowing the rotor position. We have not going into the details in this paper, but BLDCM are usually built with some rotor position sensor, commonly HallEffect sensors, that feed some position encoder [19]. This provides a high precision measure of actual rotor position and actual rotor angular speed, also needed to close the loop.

Finally, the rotor axis turns the gear to reduce the speed of the wheel axis. For the purpose of this paper, the terrain and friction effects are modeled as a constant torque by a fixed effort source. 


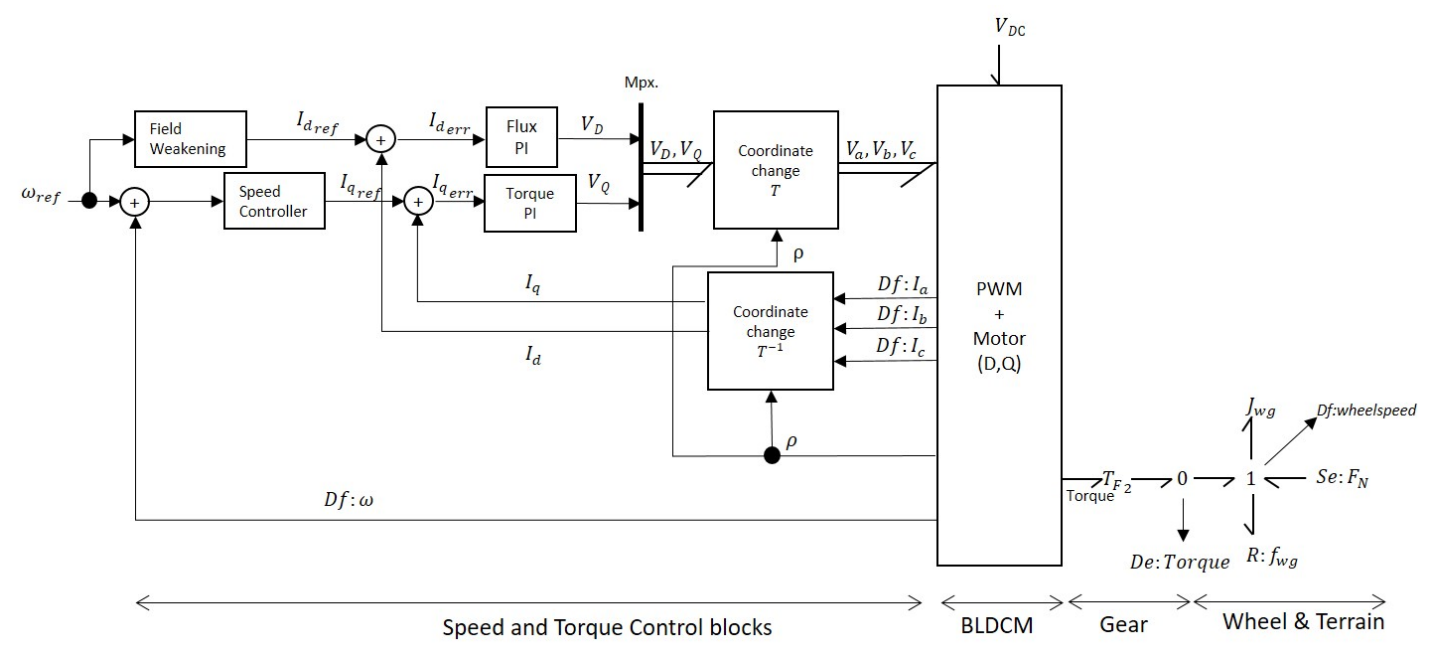

Figure 9: Traction subsystem motor-wheel Bond Graph/Block diagram.

\subsection{PWM plus BLDCM block}

Figure 7 shows the details of the BLDCM block diagrams, almost to the physical components interconnection level. The stator voltages enter the PWD module, which provides power from a DC supply, and generates the tensions that go to the stator electrical terminals. The motor provides the useful torque and the rotor position and angular speed.

Keep in mind that because the BG model of the BLDCM is given in the reference frame coordinate system, and additional transformation from $V_{a_{p w m}}, V_{b_{p w m}}$, and $V_{c_{p w m}}$ to $\left(V_{d}, V_{q}\right)$ is needed. To avoid clumping the image, this additional transformation is not included in Figure 7. This additional transformation in needed only to simulate the system. It can be seen in Figure 10, and correspond to the coordinates change stage.

Although Bond Graph models of PWM can be easily found, [13, 8, 6], we won't describe it at that fine level of detail, because we are not interested on diagnosing the PWM components. To simulate the system we employed the implementation provided in [8] which uses a triangular carrier [12].

\subsection{BLCDM block Bond Graph Model}

The BG model of the BLDCM is shown in Figure 10.

The model inputs are the actual stator voltages, $\left(V_{a}, V_{b}, V_{c}\right)$. We have preserved this standard notation to maintain the consistency with most of the literature on BLDCM. However, the reader must be aware that these variables are the triplet $\left(V_{a_{p w m}}, V_{b_{p w m}}, V_{c_{p w m}}\right)$ generated by the PWM module.

The stator voltages are first transformed to the reference frame $(d, q)$ and then injected in the BG model in reference frame of the BLDCM. Hence, Figure 10 shows the model that has to be used to simulate the motor in the Traction subsystem diagram of Figure 9. 


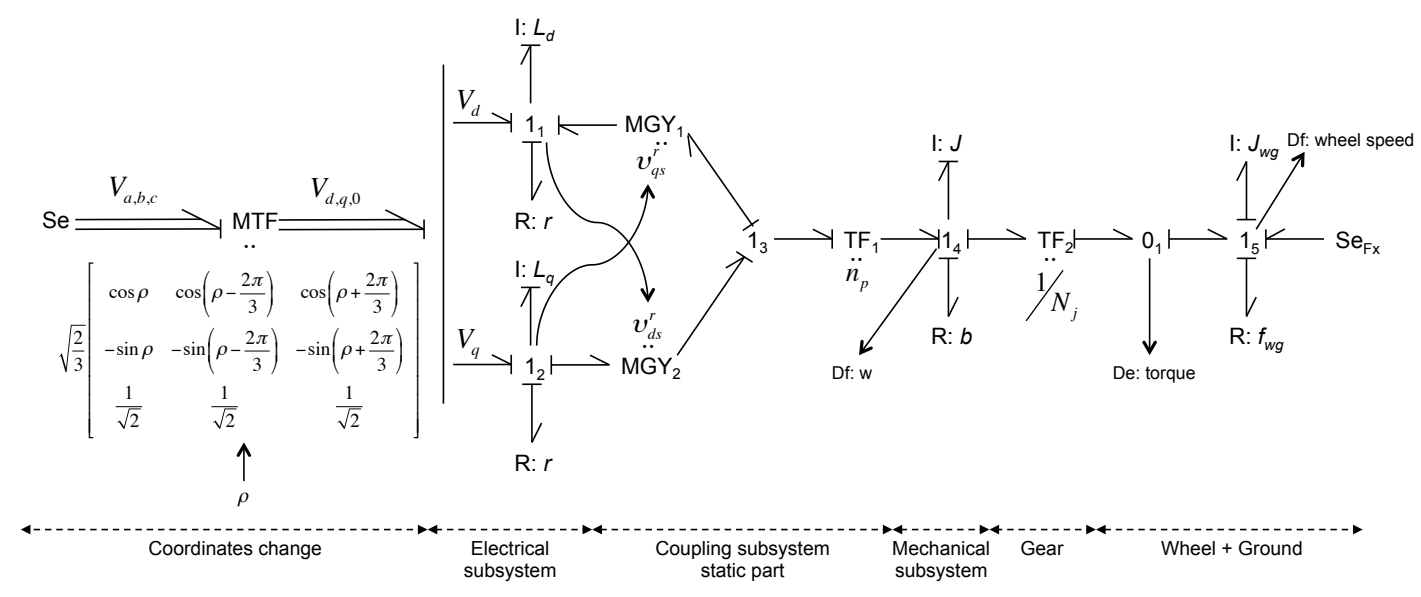

Figure 10: Alternative design with BLDCM.

\section{Bond-Graph Possible Conflicts}

The Possible Conflict, PC, approach is a dependency-compilation technique from the DX community [24], that have been successfully used for system model decomposition in consistency-based diagnosis of continuous systems. PCs define minimal structurally overdetermined subsets of equations with sufficient analytical redundancy to generate fault hypotheses from observed measurement deviations. In the original approach, only structural and causal information from the system model was used. PCs can be derived from different kinds of models: ODEs, ADEs, and Temporal Causal Graphs derived from BG models [5]. Later on were extended for diagnosis of hybrid systems through the Hybrid Bond-Graph Possible Conflicts (HBG-PCs) concept [4, 25].

Hybrid Bond Graphs (HBGs) extend Bond-Graphs by including idealized switching junctions, (Sw) to model mode changes in the system. If a SW is set to $\mathrm{ON}$, it behaves as a regular junction. When it changes to OFF, all bonds incident on the junction are deactivated forcing 0 flow (or effort) for 1 (or 0 ) junctions.

In the new HBG-PC proposal we defined first what is a BG-PC, without considering the presence of SW. Hence we can use the same approach as in HBG-PCs for computing BG-PCs directly from the HBG model because it is clear that a HBG without SW is a valid BG model. Hence the main step is to derive minimally Redundant Bond-Graphs (RBG-PCs).

Given the underlying equations for each bond in the graph, searching for RBG-PCs require two definitions outside the traditional BG modelling formalism:

- Degenerated junction: if a 0 (resp. 1) junction has a sensor that provides the value for the effort (resp. flow), we can use the dualized version of the sensor and we do not need to use the remaining bonds and components to compute that magnitude value.

- Non-parametric paths: that allow propagating flow or effort from a source of a sensor independently of passive elements (such as capacitors, resistors or inductance elements). These paths usually contains only junctions without passive elements or degenerated junctions, but can traverse 0 (respectively 1 )-junctions if effort (resp. flow) is known.

RBG-PCs are computed for each available output measurement in the system, searching backwards 
until sources or other measurements are found, using only structural information (i.e. do not taking into account possible causal assignments). If the RBG-PC has a valid causal assignment, it is a BG-PC.

BG-PCs structure and models can be computed offline. Also we can compute offline qualitative information about the influence of the faults in the residuals. Afterwards, they can be used online to track the system current mode and perform fault detection and diagnosis ${ }^{1}$. This is called the Reduced Qualitative Fault Signature Matrix (RQFSM) that provides the expected deviation in the residual for each faulty parameter in the BG-PC for the current mode.

Our approach is consistency-based and we will use the information provided in the previous matrices to discard faults whose signature is not consistent with current observations.

\section{DBM for the Traction Subsystem using BG-PCs}

\subsection{Faults in the locomotion subsystem}

For the sake of simplicity we will not consider faults neither in the controllers software nor the microelectronic components nor the PWM modules in Figures 9 and 8. We focus on faults in physical parameters in the electric and mechanical parts of the BLDCM and the gear and load (wheel and Terrain) subsystems.

Looking at the physical parameters in the BG model in figure 10 we see that we have potential faults in inductances $\left\{L_{d}, L_{q}, r\right\}$ and resistors in the stator and rotor parts: $\{r, J\}$, together with potential faults in the transformers $T F_{1}$ and $T F_{2}$. Additionally we have faults in the mechanical parts in the gear and load subsystems: $\left\{J_{w g}, f_{w g}\right\}$.

\subsection{BG-PCs in the system}

BG-PCs are found searching for minimally Redundant Bond-Graphs for each potential discrepancy node, in our system they are the output sensors: $D f: w, D e:$ Torque, and $D f:$ wheelspeed. The search will proceed always backwards until we reach a source or a sensor (usually related to degenerated junctions).

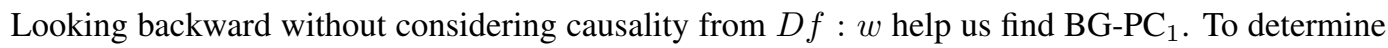
the motor speed $D f: w$ we reach junction $1_{4}$. The flow is determined by $J$ but we need the effort in that bond, hence every element is included: $J, R, T F_{1}, T F_{2}$. To determine effort in $T F_{2}$ we move to $O_{1}$ which is a degenerated junction because we measure the effort in De:Torque. There is no more propagation in that direction. Searching from $T F_{1}$ backwards we arrive to junction $1_{3}$. To determine the flow we need every bond, including $M G Y_{1}$ and $M G Y_{2}$. To determine the effort in $M G Y_{1}$ we need junctions $1_{1}$ and $1_{2}$. To determine the effort in them we need all the elements: $\left\{L_{d}, R: r, V_{d}\right\}$. Symmetrically, we include all the remaining elements for $M G Y_{2}$, adding $L_{q}$ and $V_{q}$. That is, we use the complete electrical and mechanical subsystems of the BLDCM, as expected. This system has a valid causal assignment, in integral causality, for every bond in the system and has minimal redundancy. It is then BG-PC 1 and its scheme can be seen in Figure 11.

Starting from De : Torque we look backwards for an effort and we reach junction $0_{1}$. The effort can be computed in junction $1_{5}$ by adding up every effort in $1_{5}$, which includes bonds $J_{w g}, f_{w g}$, and $S e: f_{x}$. To compute the efforts we need the flow, provided by the sensor $D f:$ wheelspeed. The search stops. The subsystem has only valid causal assignment if we use derivative causality in $J_{w g}$. This can lead to computational problems, but that depends on the simulation software. The $\mathrm{BG}-\mathrm{PC}_{2}$ can be seen in Figure 12.

\footnotetext{
${ }^{1}$ Usually diagnosing sensor faults is easier than diagnosing these later faults, we have decided to center only on parametric and discrete faults from now on.
} 


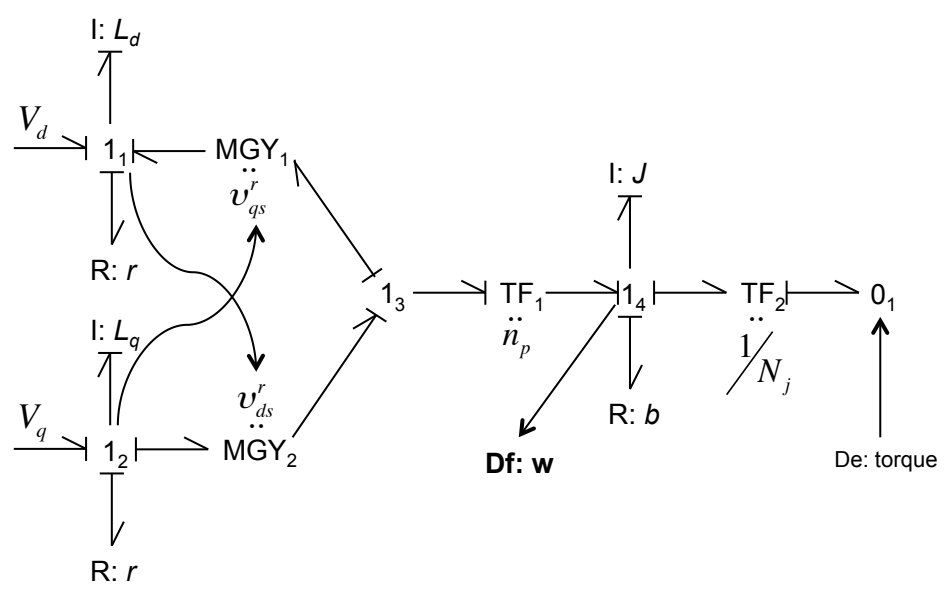

Figure 11: BG-PC 1 .

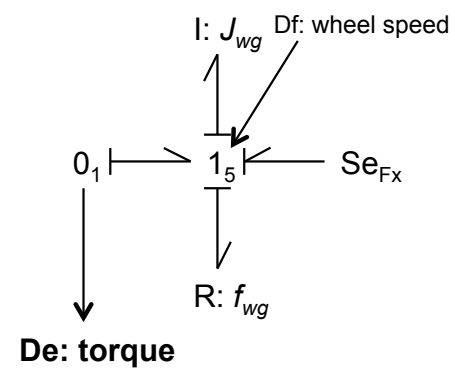

Figure 12: $\mathrm{BG}-\mathrm{PC}_{2}$.

Starting from $D f:$ wheelspeed we search backwards to $1_{5}$ looking for a flow variable. Hence we include all elements and proceed looking for new junctions, reaching $O_{1}$. We can not use De:Torque because it is a effort, and we are looking for a flow, hence include in $T F_{2}$, then $1_{4}$ which is a degenerated

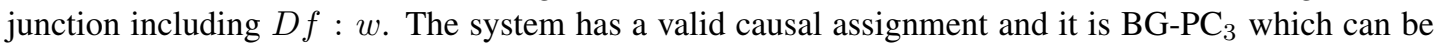
seen in 13.

\subsection{Detectability and Isolability properties}

The detection capabilities in the set of BG-PCs can be extracted from the theoretical fault signature matrix of the system, shown in Table1.

For the given sensors, speeds and torque, the system is fully detectable. However, fault isolability of the system is almost null, with only a fault in the gear been fully isolable form the rest. In particular, no single motor fault is isolable, because all of them occur in the same conflict.

The isolability of the system may increase if we assume that the stator current measurements are available, which is not always the case, even though they are needed to close the control loop. 


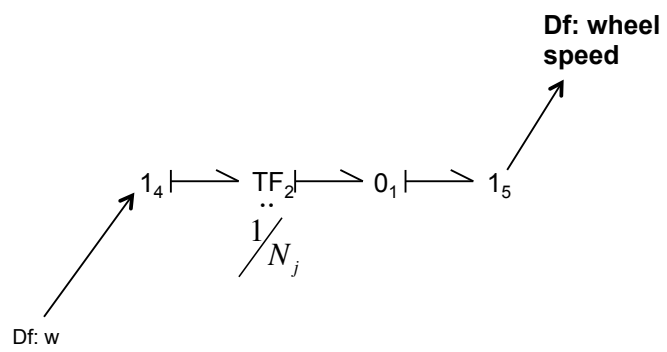

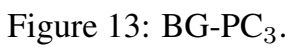

\begin{tabular}{|c|c|c|c|c|c|c|c|c|c|}
\hline BG-PC & $L_{d}$ & $L_{q}$ & $r$ & $T F_{1}$ & $J$ & $b$ & $T F_{2}$ & $J_{w g}$ & $f_{w g}$ \\
\hline BG-PC $_{1}$ & 1 & 1 & 1 & 1 & 1 & 1 & 1 & 0 & 0 \\
\hline BG-PC $_{2}$ & 0 & 0 & 0 & 0 & 0 & 0 & 0 & 1 & 1 \\
\hline BG-PC $_{3}$ & 0 & 0 & 0 & 0 & 0 & 0 & 1 & 0 & 0 \\
\hline
\end{tabular}

Table 1: Fault localization capabilities for the set of BG-PCs.

\section{Simulation Results}

We have simulated the complete system on the tool 20-sim, [8], a graphical oriented Bond-Graph modeling and simulation tool. We have parametrized the motor to match Maxon EC-4pole 22 brushless motor, part number 323218 [20]. PI controllers were manually adjusted.

To test the validity of the model we have simulated the system under nominal load and speed, checking that the system response to speed set point changes is admissible.

\subsection{Testing in several diagnosis scenarios}

To illustrate the validity of the BG-PCs models we have tested several diagnosis scenarios in simulation related to the motor and the mechanical part that connects the motor and the traction system (gear, wheel), hence we only provide simulation for the model $B G_{-} P C 1$ given a fixed input torque.

We show in the paper two different fault scenarios. The first one correspond to an increase in the motor axe bearings friction, than can be simulated increasing the value of the resistance connected to union $1_{4}$. The second one correspond to an electrical failure of the stator coils, which can be modeled modifying both the inductance and the electrical resistance of the electrical subsystem mode.

We have simulated several scenarios for 2 seconds, having a sampling period of 0.01 seconds. All the simulations start from a stationary state and we introduce a $5 \%$ change in the speed set point at $t=1 \mathrm{~s}$. Later on we introduce the fault at $t=1.2 \mathrm{~s}$. It can be seen in the first $1.2 \mathrm{~s}$ of each experiment that the system is capable to reach a new stationary state without any influence in the residual, hence due to lack of space we do not provide a simulation for the nominal, i.e. non-faulty, scenario.

Figure 14 shows the evolution of the $D f:$ wheelspeed signal (in rpm) for a fault in $R: b$. The size of the fault is $5 \%$. We can see that the residual is almost zero before the fault occurrence and there is a clear activation soon after the injection of the fault at $t=1.2 \mathrm{~s}$.

Figure 15 shows the evolution of the $D f: w$ signal (in rpm) for a fault in the motor. The fault has been simulated as a lack of performance in all the parameters of the motor. This is necessary because of the simulation model in DQ coordinates. The size of the fault is $5 \%$. We can see that the residual is 

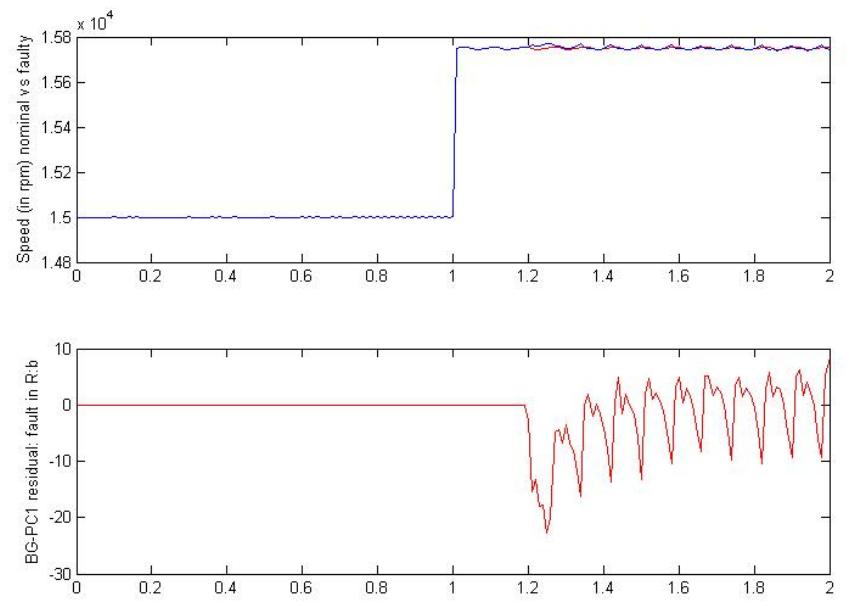

Figure 14: Fault scenario 1: fault in Resitance $R: b$.

almost zero before the fault occurrence and there is a clear activation soon after the injection of the fault at $t=1.2 s$.
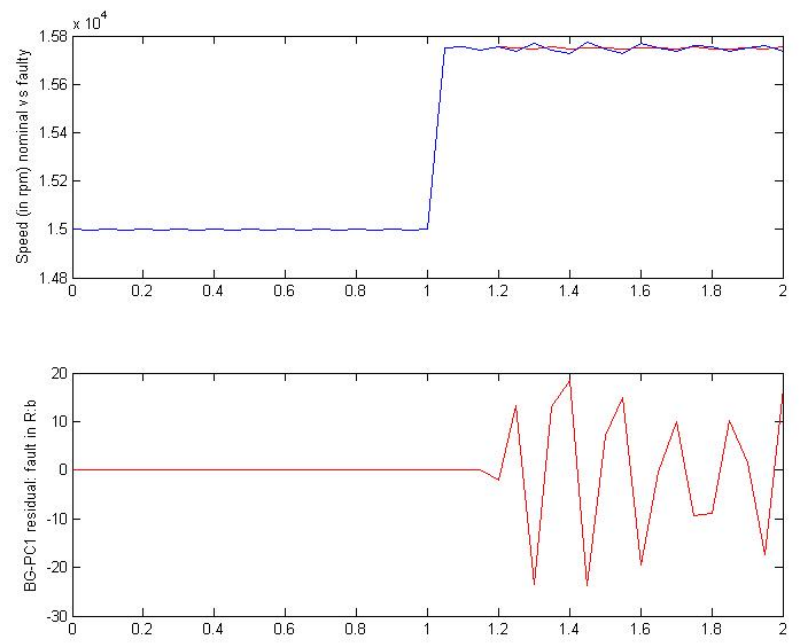

Figure 15: Fault scenario 1: fault in the motor.

In both fault scenarios can be also clearly seen the influence of the control loops in the residual values. 


\section{Conclusions}

Autonomous vehicles, and more specifically rovers for planetary explorations, demand automated diagnosis to help detect faults and try to overcome their effects on the whole system. Traction subsystem are fundamental parts in these vehicles. In this work we have proposed how to change existing design for a mars lunar rover [17] to include a more efficient and less energy demanded type of motor: brushless DC engines.

There are several proposals in the literature about these type of engines, but it is necessary to adapt the controllers to generate appropriate control actions. This work propose to adapt existing solutions for the control of these systems [13], and to couple it with a generic type of traction subsystem [15, 18]. The main contribution of this work is to provide a unique modelling framework for both the simulation and diagnosis of these types of systems: the BG models [6,26] and Bond-Graph Possible Conflicts, a simplified version of Hybrid Bond-Graph Possible Conflicts [4, 25].

In this work we have proposed a detailed description of the Bond-Graph subsystems required for the traction subsystem and the set of BG-PCs that we can obtain from the BG model of the engine and the mechanical part of the subsystem. This proposal poses a new challenge because we need to develop simulation models capable to work well under integral and derivative causality. This has not been done yet, to the best of our knowledge.

This is ongoing work and we are testing faults and diagnosis scenarios, together with a complete diagnosability analysis. In this paper we have only tested the validity of the approach under a minimum number of sensors. As further research we want to explore a more general hybrid and distributed architecture capable to diagnose faults in the whole traction system, including the controller and the four/six wheel engines.

In this work we have opted by one possible control configuration; allowing different configurations will lead to an hybrid system. This is our current research topic right now, extending the current BG-PC approach with Hybrid Bond-Graph Possible Conflicts [4, 22, 25].

\section{References}

[1] Edward Balaban, Sriram Narasimhan, Matthew Daigle, José Celaya, Indranil Roychoudhury, Bhaskar Saha, Sankalita Saha, and Kai Goebel. A mobile robot testbed for prognostics-enabled autonomous decision making. In Annual conference of the prognostics and health management society, pages 15-30. PHM Society Rochester, NY, 2011.

[2] Edward Balaban, Sriram Narasimhan, Matthew Daigle, Indranil Roychoudhury, Adam Sweet, Christopher Bond, and G Gorospe. Development of a mobile robot test platform and methods for validation of prognosticsenabled decision making algorithms. International Journal of Prognostics and Health Management, 4(1), 2013.

[3] R Giridhar Balakrishna and P Yogananda Reddy. Speed control of brushless DC motor using microcontroller. International Journal of Engineering Technology, Management and Applied Sciences, 3(6):11-26, 2015.

[4] A. Bregon, C. Alonso, G. Biswas, B. Pulido, and N. Moya. Fault Diagnosis in Hybrid Systems using Possible Conficts. In Proc. of the IFAC SAFEPROCESS'12, Mexico D.F., Mexico, 2012.

[5] A. Bregon, G. Biswas, and B. Pulido. A Decomposition Method for Nonlinear Parameter Estimation in TRANSCEND. IEEE Trans. Syst. Man, Cyber. Part A, 42(3):751-763, 2012.

[6] Jan F Broenink. 20-sim software for hierarchical bond-graph/block-diagram models. Simulation Practice and Theory, 7(5):481-492, 1999.

[7] J.F. Broenink. Introduction to Physical Systems Modelling with Bond Graphs. SiE Whitebook on Simulation methodologies, 1999.

[8] Controllab Products B.V. 20sim. http://www.20sim.com/. 
[9] Copley Controls Corp. Field oriented control and what good is it? Technical Report http://www.copleycontrols.com/Motion/pdf/Field-Oriented-Control/, 2002.

[10] Cypress Semiconductors. Field weakened implementation pmsm drive fm3 microcontroller. http://www.cypress.com/file/224021/download.

[11] Alexandros Frantzis Gounaris, Pantelis Poulakis, Christophe Chautems, Carloni Raffaela, and Stefano Stramigioli. Lunar rover model-reengineering of an existing mobile platform towards the realization of a rover autonomy testbed. 2011.

[12] Joachim Holtz. Pulsewidth modulation for electronic power conversion. Proceedings of the IEEE, 82(8):1194-1214, 1994.

[13] Sergio Junco and Alejandro Donaire. Bond Graph modeling and simulation of electrical machines. In Bond Graph Modelling of Engineering Systems, pages 269-321. Springer, 2011.

[14] Paul Krause, Oleg Wasynczuk, Scott D Sudhoff, and Steven Pekarek. Analysis of electric machinery and drive systems, volume 75. John Wiley \& Sons, 2013.

[15] Rui Loureiro, Rochdi Merzouki, and Belkacem Ould Bouamama. Structural reconfiguration conditions based on Bond Graph approach: application to an intelligent autonomous vehicle. IFAC Proceedings, 45(20):970 975, 2012.

[16] A. Medina, G. Binet, and P. Colmenarejo. An Integrated Autonomous Navigation and Decision-Making Architecture for Planetary Exploration Rovers, pages 97-112. Springer International Publishing, 2014.

[17] Alberto Medina, Luis Mollinedo, Konstantinos Kapellos, and Pantelis Poulakis. Design and realization of a rover autonomy testbed. In Procs. of ASTRA 2015 - 13th ESA Workshop on Advanced Space Technologies for Robotics and Automation, Noordwijk, The Netherlands, may 2105.

[18] Rochdi Merzouki, Arun K Samantaray, Pushparaj Mani Pathak, and Belkacem Ould Bouamama. Intelligent mechatronic systems: Modeling, control and diagnosis. Springer Science \& Business Media, 2012.

[19] Maxon motors AG. Motor control for newbies. http://www.maxonmotor.com/medias/sys_master/root/ 8812125782046/MotionControlforNewbies.pdf, Switzerland.

[20] Maxon motors AG. Motor ec4 specifications. http://docs-europe.electrocomponents.com/webdocs/0f0a/0900766b80f0a497.pdf, Switzerland.

[21] Maxon motors AG. Suddenly, everyone wants to go to the moon. http://www.maxonmotor.com/maxon/view/application/, Switzerland.

[22] Noemi Moya, Anibal Bregon, Carlos J. Alonso-González, and Belarmino Pulido. A common framework for fault diagnosis of parametric and discrete faults using Possible Conflicts. In Advances in Artificial Intelligence: 15th Conference of the Spanish Association for Artificial Intelligence, CAEPIA 2013, Madrid, Spain, September 17-20, 2013. Proceedings, pages 239-249, Berlin, Heidelberg, 2013. Springer Berlin Heidelberg.

[23] S. Narasimhan and G. Biswas. Model-Based Diagnosis of Hybrid Systems. IEEE Trans Syst Man Cyber Part A, 37(3):348-361, May 2007.

[24] B. Pulido and C. Alonso-González. Possible Conflicts: A compilation technique for consistency-based diagnosis. IEEE Trans Syst Man Cyber Part B, 34(5):2192-2206, Oct. 2004.

[25] Belarmino Pulido, Carlos Alonso-González, Anibal Bregon, and Alberto Hernández. Characterizing and computing HBG-PCs for hybrid systems fault diagnosis. In Conference of the Spanish Association for Artificial Intelligence, pages 116-127. Springer, 2015.

[26] A. K. Samantaray and B. O. Bouamama. Model-based Process Supervision: A Bond Graph Approach. Springer-Verlag, London, 2008.

[27] V.Sowmya Sree and N. Ravi Sankar Reddy. Application of Bond Graph to permanent magnet brushless DC motor drive. Journal of Electrical Engineering, 15:148-154, 2015.

[28] Youcef Touati, Rochdi Merzouki, Belkacem Ould Bouamama, and Rui Loureiro. Detectability and isolability conditions in presence of measurement and parameter uncertainties using bond graph. In The 8th International Federation of Automatic Control (IFAC) Symposium SAFEPROCESS-2012, page 6, 2012. 